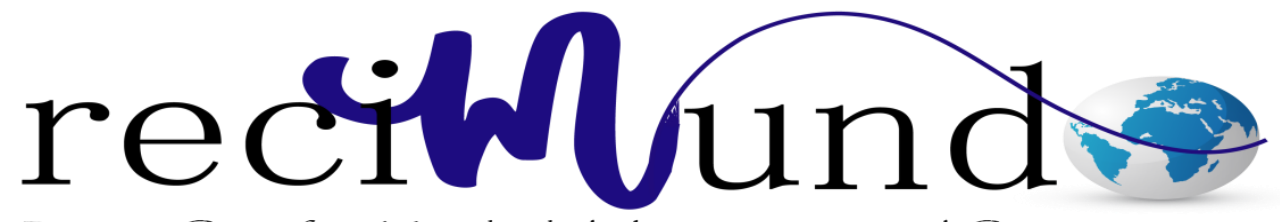

Revista Científica Mundo de la Investigación y el Conocimiento

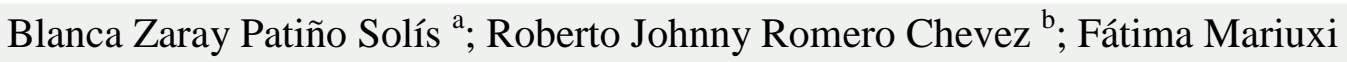
Conto Díaz ${ }^{c}$; Jenny Leonor Pincay Criollo ${ }^{\mathrm{d}}$

Prevalencia de enfermedad periodontal en pacientes que usen prótesis dentales parciales

Revista Científica Mundo de la Investigación y el Conocimiento. Vol. 2 núm.2, mayo, ISSN: 2588-073X, 2018, pp. 356-367

DOI: 10.26820/recimundo/2.(2).2018.356-367

Editorial Saberes del Conocimiento

Recibido: 15/12/2017

Aceptado: $25 / 03 / 2018$

a. Universidad de Guayaquil; caritadeluna-1993@hotmail.es

b. Universidad de Guayaquil; roberto.romeroch@ug.edu.ec

c. Universidad de Guayaquil; fatima.contod@ug.edu.ec

d. Universidad de Guayaquil; jenny.pincayc@ug.edu.ec 


\section{Prevalencia de enfermedad periodontal en pacientes que usen prótesis \\ dentales parciales}

Vol. 2, núm. 2., (2018)

Blanca Zaray Patiño Solís; Roberto Johnny Romero Chevez; Fátima Mariuxi Conto Díaz; Jenny

Leonor Pincay Criollo

\section{RESUMEN}

A lo largo de la carrera odontológica hemos visto muchos casos de enfermedad periodontal en pacientes que usan prótesis parciales removibles esto se ha dado por muchas causas como mala higiene bucal, material de las prótesis, o simplemente pacientes que no cuidan sus dientes lo cual ha provocado que dichos pacientes sufran desde una simple gingivitis hasta una periodontitis grave esto es debido a que las bacterias que se encuentran dentro de la cavidad bucal producen factores de viruela esto quiere decir que se propagan provocando así la fase inicial o placa bacteriana; el objetivo de nuestro estudio fue establecer la prevalencia de enfermedad periodontal en pacientes que usan prótesis parciales removibles a través de encuestas a diferentes profesionales; De dicha investigación hemos obtenido como resultado que del total de la muestra de estudio de 80 pacientes con prótesis parcial removible se diagnosticaron 50 pacientes con enfermedad periodontal vinculado al uso de P.P.R. (70\%) y 30 no presentaron (30\%), De los 50 pacientes con enfermedad periodontal que usan prótesis parcial removible se tuvo como respuesta que 35 pacientes pertenecían al género masculino (70\%), y 15 pacientes pertenecían al género femenino género femenino (30\%) y del total de la muestra de estudio de 50 pacientes con enfermedad periodontal que usan prótesis parcial removible se tuvo como respuesta que 20 pacientes pertenecían al grupo etareo de 30 a 40 años de edad (40\%), y 15 pacientes pertenecían al grupo etareo de 41 a 70 años de edad (60\%); Frente a la investigación realizada llegamos a la conclusión de que el género masculino es el más propenso a sufrir enfermedad periodontal y las edades donde más se presenta es ente los 41 a 70 años por lo tanto es donde se debe tener mayor precaución.

Palabras clave: Prótesis parcial removible, placa bacteriana, enfermedad periodontal, cuidado bucal. 


\section{Prevalencia de enfermedad periodontal en pacientes que usen prótesis dentales parciales}

Vol. 2, núm. 2., (2018)

Blanca Zaray Patiño Solís; Roberto Johnny Romero Chevez; Fátima Mariuxi Conto Díaz; Jenny Leonor Pincay Criollo

\section{ABSTRACT}

Throughout the dental career we have seen many cases of periodontal disease in patients using removable partial dentures that have been given for many reasons such as poor oral hygiene, material prostheses, or just patients who do not take care of their teeth which has caused such patients suffer from simple gingivitis to severe periodontitis this is because the bacteria found in the oral cavity smallpox produce factors that means are spread thereby causing the initial phase or plaque; The aim of our study was to establish the prevalence of periodontal disease in patients using removable partial dentures through surveys different professionals; In this investigation we have obtained the result that the total study sample of 80 patients with removable partial denture 50 patients were diagnosed with periodontal disease linked to the use of P.P.R. (70\%) and 30 not present $(30 \%)$ of the 50 patients with periodontal disease using removable partial denture was in response to 35 patients were male gender (70\%), and 15 patients were female female (30\%) and the total study sample of 50 patients with periodontal disease using removable partial denture was in response to 20 patients were in the age group 30 to 40 years old (40\%), and 15 patients were etareo the group of 41-70 years (60\%); Faced with the research we concluded that male gender is more prone to periodontal disease and age where it is being presented 41 to 70 years hence is where you should be more careful.

Key words: Removable partial dentures, plaque, periodontal disease, oral car. 


\section{Prevalencia de enfermedad periodontal en pacientes que usen prótesis \\ dentales parciales}

Vol. 2, núm. 2., (2018)

Blanca Zaray Patiño Solís; Roberto Johnny Romero Chevez; Fátima Mariuxi Conto Díaz; Jenny

Leonor Pincay Criollo

\section{Introducción.}

El principal objetivo del tratamiento con prótesis parcial removible (PPR), además de reponer las estructuras pérdidas, es preservar y proteger las estructuras remanentes. Sin embargo, hace 25 años, existía una opinión generalizada de que la PPR, especialmente aquella de extremo libre, estaría frecuentemente asociada con caries y enfermedad periodontal. Este pensamiento se basaba en estudios que mostraban equivocadamente un efecto negativo de este tipo de prótesis sobre los dientes y el periodonto. (Sánchez, Andrés, \& Troconis, 1999)

El avance científico, especialmente en el área de Periodoncia, tuvo como consenso que la biopelícula dental es la principal, y probablemente, única responsable de la gingivitis y periodontitis, esto estimuló a los protesistas a aplicar este nuevo conocimiento en el mantenimiento de sus tratamientos. Así, hoy se sabe que el éxito de la rehabilitación con PPR está directamente relacionado a la importancia dada a la higiene oral y controles periódicos, ya que la presencia de dicho aparato en boca aumenta la posibilidad de adhesión de la biopelícula dental, lo cual exige mayores cuidados en la higiene bucal. (Farias Neto, Giancarlo, \& Porto Carreiro, 2013)

Con la introducción de la Odontología Preventiva, en la mitad del siglo XX, se pudo percibir que los dientes naturales pueden ser mantenidos en boca por toda la vida de los individuos. En los Estados Unidos, por ejemplo, se observó en las últimas décadas un descenso constante en la prevalencia de la pérdida dentaria, con un número creciente de pacientes reteniendo más dientes en boca y por un periodo mayor. Lo mismo es esperado en países europeos en un futuro cercano. (Esquivel Hernández \& Jiménez Férez, 2012) 


\section{Prevalencia de enfermedad periodontal en pacientes que usen prótesis dentales parciales}

Vol. 2, núm. 2., (2018)

Blanca Zaray Patiño Solís; Roberto Johnny Romero Chevez; Fátima Mariuxi Conto Díaz; Jenny Leonor Pincay Criollo

Sumándose al bien evidenciado descenso de la pérdida dentaria, en los últimos años, fue posible observar el éxito de la terapia con implantes óseointegrados, lo que posibilitó la creación de un contexto en el cual se puede cuestionar el futuro de los procedimientos protéticos. Este tema es de interés para toda la comunidad odontológica, pues está directamente relacionado a la necesidad y demanda por tratamiento clínico, así como a su enseñanza en las universidades. (Cáceres, Noriega, \& Coz, 2012) Así el presente artículo busca discutir la importancia de la Prótesis Parcial Removible en el contexto de la Odontología actual, basándose en los cambios ocurridos en las últimas décadas. (Fuentes \& Oporto, 2015)

El objetivo de la presente investigación fue establecer la prevalencia de enfermedad periodontal en pacientes que usan prótesis parcial removible en la Ciudad de Guayaquil, parroquia Ximena pacientes de 30 a 70 años de edad durante el año 2015.

\section{Metodología.}

\section{Diseño y tipo de investigación}

\section{Diseño de investigación}

El diseño de investigación es cuantitativa, descriptiva analítica.

Investigación cuantitativa porque midió y evaluó el nivel de conocimientos de los pediatras y padres de familia sobre medidas preventivas en salud bucal.

Investigación descriptiva: se describieron los resultados obtenidos de las encuestas al evaluar dos tipos de sujetos diferentes pediatras y padres de familia. 


\section{Prevalencia de enfermedad periodontal en pacientes que usen prótesis \\ dentales parciales}

Vol. 2, núm. 2., (2018)

Blanca Zaray Patiño Solís; Roberto Johnny Romero Chevez; Fátima Mariuxi Conto Díaz; Jenny

Leonor Pincay Criollo

Investigación analítica: y se realizó la comparación de variables entre los grupos objeto de estudio que fueron los pediatras y los padres de familia.

El presente trabajo es de diseño Cuantitativo ya que vamos analizar los datos recopilados en cantidades numéricas de la clínica Servident S.A. a través de una encuesta que realizaremos a cada uno de los especialistas para después proceder al conteo y análisis de las respuestas obtenidas.

Tipo de investigación

Esta investigación es de tipo exploratoria, descriptiva y de campo

Investigación Exploratoria: Es aquella que se efectúa sobre un tema u objeto desconocido o poco estudiado, por lo que sus resultados constituyen una visión aproximada de dicho objeto, es decir, un nivel superficial de conocimiento. Los estudios exploratorios se efectúan, normalmente, cuando el objetivo es examinar un tema o problema de investigación poco estudiado o que no ha sido abordado antes. Los estudios exploratorios en pocas ocasiones constituyen un fin en sí mismos, por lo general determinan tendencias, identifican relaciones potenciales entre variables y establecen el 'tono' de investigaciones posteriores más rigurosas". (DANKHE, 1986)

Investigación descriptiva: Los estudios descriptivos buscan especificar las propiedades importantes de personas, grupos, comunidades o cualquier otro fenómeno que sea sometido a análisis. Miden y evalúan diversos aspectos, dimensiones o componentes del fenómeno o fenómenos a investigar. Desde el punto de vista científico, describir es medir. Esto es, en un 


\section{Prevalencia de enfermedad periodontal en pacientes que usen prótesis dentales parciales}

Vol. 2, núm. 2., (2018)

Blanca Zaray Patiño Solís; Roberto Johnny Romero Chevez; Fátima Mariuxi Conto Díaz; Jenny Leonor Pincay Criollo

estudio descriptivo se selecciona una serie de cuestiones y se mide cada una de ellas independientemente, para así y valga la redundancia describir lo que se investiga. Tamayo (1991) precisa que: "la investigación descriptiva comprende la descripción, registro, análisis e interpretación de la naturaleza actual, composición o procesos de los fenómenos”. (DANKHE, 1986)

Investigación de campo es: El análisis sistemático de problemas en la realidad, con el propósito bien sea de describirlos, interpretarlos, entender su naturaleza y factores constituyentes, explicar sus causas y efectos, o predecir su ocurrencia, haciendo uso de métodos característicos de cualquiera de los paradigmas o enfoques de investigación conocidos o en desarrollo. Los datos de interés son recogidos en forma directa de la realidad; en este sentido se trata de investigaciones a partir de datos originales o primarios. (DANKHE, 1986)

\section{Población y muestra}

El presente trabajo se realizó en un numero de 80 pacientes en los cuales realizamos un diagnostico con ayuda de los odontólogos de la clínica Servident S.A.

El tipo de muestreo a utilizar es no probabilístico debido a que la población es pequeña, por lo tanto se tomara en cuenta todo el grupo encuestado. 


\section{Prevalencia de enfermedad periodontal en pacientes que usen prótesis \\ dentales parciales}

Vol. 2, núm. 2., (2018)

Blanca Zaray Patiño Solís; Roberto Johnny Romero Chevez; Fátima Mariuxi Conto Díaz; Jenny

Leonor Pincay Criollo

Métodos técnicas e instrumentos

El método de trabajo utilizado es inductivo-deductivo debido a que con la población encuestada y a los antecedentes redactados realizaremos las conclusiones y resultados de nuestra investigación.

- La técnica empleada para esta investigación es a través de encuestas individuales.

- Los instrumentos a utilizar son: papel, laptop, esferográficos, carpetas, impresora, clips, lápices, correctores, auto, cámara fotográfica.

Procedimiento de la investigación

Para realizar nuestra investigación procedimos a solicitar el permiso en la clínica odontológica Servident S.A. luego procedimos analizar artículos científicos relacionados al tema de investigación, después observamos a cada uno de los pacientes y les realizamos su respectivo diagnóstico, como siguiente paso procedemos a reunir todos los datos obtenidos, después evaluaremos los resultados elaboraremos las tablas y gráficos, luego realizaremos las conclusiones y recomendaciones. 
Prevalencia de enfermedad periodontal en pacientes que usen prótesis dentales parciales

Vol. 2, núm. 2., (2018)

Blanca Zaray Patiño Solís; Roberto Johnny Romero Chevez; Fátima Mariuxi Conto Díaz; Jenny Leonor Pincay Criollo

\section{Resultados.}

Tabla \# 1.- Distribución de los pacientes con enfermedad periodontal que usan prótesis parcia removible

\begin{tabular}{|c|c|c|}
\hline $\begin{array}{l}\text { Enfermedad } \\
\text { Periodontal }\end{array}$ & FRECUENCIA & PORCENTAJE $(\%)$ \\
\hline Si & 50 & $60 \%$ \\
\hline No & 30 & $40 \%$ \\
\hline Total & 80 & $100 \%$ \\
\hline
\end{tabular}

Del total de la muestra de estudio de 80 pacientes con prótesis parcial removible se diagnosticaron 50 pacientes con enfermedad periodontal vinculado al uso de P.P.R. (70\%) y 30 no presentaron $(30 \%)$.

Tabla \#2.- Distribución de los pacientes con enfermedad periodontal que usan prótesis parcia removible según su género

\begin{tabular}{|c|c|c|}
\hline GERNERO & FRECUENCIA & PORCENTAJE $(\%)$ \\
\hline Masculino & 35 & $70 \%$ \\
\hline Femenino & 15 & $30 \%$ \\
\hline Total & 50 & $100 \%$ \\
\hline
\end{tabular}




\section{Prevalencia de enfermedad periodontal en pacientes que usen prótesis \\ dentales parciales}

Vol. 2, núm. 2., (2018)

Blanca Zaray Patiño Solís; Roberto Johnny Romero Chevez; Fátima Mariuxi Conto Díaz; Jenny

Leonor Pincay Criollo

Del total de la muestra de estudio de 50 pacientes con enfermedad periodontal que usan prótesis parcial removible se tuvo como respuesta que 35 pacientes pertenecían al género masculino (70\%), y 15 pacientes pertenecían al género femenino género femenino (30\%).

Tabla \#3.- Distribución de los pacientes con enfermedad periodontal que usan prótesis parcia removible según su edad

\begin{tabular}{|c|c|c|}
\hline Edades & FRECUENCIA \\
\hline $\mathbf{3 0}-\mathbf{5 0}$ años & 20 & $40 \%$ \\
\hline $\mathbf{5 1 - 7 0}$ años & 30 & $60 \%$ \\
\hline Total & 50 & $100 \%$ \\
\hline
\end{tabular}

Del total de la muestra de estudio de 50 pacientes con enfermedad periodontal que usan prótesis parcial removible se tuvo como respuesta que 20 pacientes pertenecían al grupo etareo de 30 a 40 años de edad (40\%), y 15 pacientes pertenecían al grupo etareo de 41 a 70 años de edad $(60 \%)$.

\section{Discusión.}

Entre las respuestas obtenidas atreves de los análisis de los casos tenemos como resultado que el género masculino es el más propenso a padecer de enfermedad periodontal esto se da por diversas razones entre una de ellas es la mala higiene bucal y o asistir a sus citas subsecuentes donde su odontólogo para que se le realice una profilaxis también hay que tener en cuenta que 


\section{Prevalencia de enfermedad periodontal en pacientes que usen prótesis dentales parciales}

Vol. 2, núm. 2., (2018)

Blanca Zaray Patiño Solís; Roberto Johnny Romero Chevez; Fátima Mariuxi Conto Díaz; Jenny Leonor Pincay Criollo

estos pacientes que padecen de enfermedad periodontal van de entre los 41 y los 70 años de edad; haciendo una comparación en investigaciones similares anteriores podemos observar que los resultados son casi similares teniendo como una de las coincidencias que el género masculino es el que más sufre de enfermedad periodontal y tiende a perder sus piezas dentarias en una edad promedio de 35 a 45 años; tendríamos como conflicto que no se estaría insistiendo en dicho genero el cuidado en su cavidad oral.

\section{Conclusiones.}

Luego del análisis de las respuestas dadas por los encuestados llegamos a las siguientes conclusiones:

El género masculino es el más propenso a sufrir enfermedad periodontal.

Uno de los mayores padecimientos a partir del uso de PPR es la gingivitis.

Los Odontólogos recomiendan que los pacientes se deben realizar profilaxis cada 6 meses.

Se tiene como principal respuesta que se debería cambiar las prótesis cada 2 años.

Una de las principales causas de enfermedad periodontal en pacientes que usan PPR es la mala higiene bucal.

Uno de los causantes de enfermedad periodontal es el metal utilizado para realizar las PPR. 


\section{Prevalencia de enfermedad periodontal en pacientes que usen prótesis \\ dentales parciales}

Vol. 2, núm. 2., (2018)

Blanca Zaray Patiño Solís; Roberto Johnny Romero Chevez; Fátima Mariuxi Conto Díaz; Jenny

Leonor Pincay Criollo

Los odontólogos dicen que a partir de los 35 a 45 años comienzan sus pacientes a utilizar PPR.

Se refiere que la edad en que los pacientes a partir de los 45 a 55 años presentan enfermedad periodontal y usan PPR.

\section{Bibliografía.}

Cáceres, A., Noriega, J., \& Coz, M. (2012). Evaluación clínico-radiográfica del éxito y supervivencia de implantes dentales con tratamiento de superficie e implantes con superficie torneada. Acta Odontológica Venezolana, 50(3).

DANKHE, O. (1986). Investigación y comunicación. México: McGrawHill.

Esquivel Hernández, R., \& Jiménez Férez, J. (2012). Efecto de la utilización de prótesis dentales en la percepción de salud bucal. REVISTA ADM, 21(2), 69-75.

Farias Neto, A., Giancarlo, D., \& Porto Carreiro, A. (2013). La prótesis parcial removible en el contexto de la odontología actual. Acta Odontológica Venezolana, 51(2).

Fuentes, F., \& Oporto, V. (2015). Impacto de la caries dental y enfermedad periodontal sobre la sexualidad de la mujer. Avances en Odontoestomatología, 31(4), 273-281.

Sánchez, Y., Andrés, E., \& Troconis, Z. (1999). La prótesis parcial removible en la práctica odontológica de Caracas, Venezuela. Acta Odontol. Venez, 37(3), 123-35. 